\title{
Closed Environment Design of Solar Collector Trough using Lenses and
} Reflectors

\author{
Kazy Fayeen Shariar ${ }^{1, *}$, Enaiyat Ghani Ovy ${ }^{2}$, Tabassum Aziz Hossainy ${ }^{2}$ \\ ${ }^{1}$ Department of Electrical \& Electronic Engineering \\ Islamic University of Technology, Board Bazar, Gazipur-1704, BANGLADESH \\ ${ }^{2}$ Department of Mechanical \& Chemical Engineering \\ Islamic University of Technology, Board Bazar, Gazipur-1704, BANGLADESH \\ *Corresponding author. Tel: +8801713083079, Fax: 880-2-9291260, E-mail: eshankonerbaiu@yahoo.com
}

\begin{abstract}
Concentrated sunlight has been used to perform useful tasks from ancient time. There are number of varieties of collector trough being used to perform these tasks. Each Varity comes with advantages as well as disadvantages. The aim of this research project is to improve the design of collector trough in terms of efficiency, lessen the heat declination, and eliminate the sun tracker mechanism. Most of the existing solar concentrators use open type trough which cause the rapid heat declination. The design attempted in this paper is to lessen this rapid declination and improve the efficiency by introducing Closed Environment Collector Trough (CECT). The CECT consists of spherical collector trough having a reflective bottom surface, five evenly distributed lenses, $30^{\circ}$ apart from each other, on the upper half of the sphere to eliminate the sun tracker and hexagonal glasses to make the environment closed and impose greenhouse effect on the system. The CECT acts like a heat trap and keeps the heat inside the chamber for a longer period of time which basically lessens the heat declination. The reflective surface and lenses concentrate the sun light directly on the fluid pipe. In this paper novel design has been proposed to improve the overall performance of solar collector troughs.
\end{abstract}

Keywords: Solar Thermal Collector, Concentrated Solar Power, Closed Environment Collector Trough.

\section{Nomenclature}

$A_{a}=$ absorber area

$\left(m^{2}\right)$

$A_{f}=$ collector geometric factor

$A_{r}=$ receiver area. $\left(m^{2}\right)$

$D_{s}=$ direct irradiation. $(W / m 2)$

$f_{w}=$ wind or exposure factor

$f_{c}=$ construction type or quality factor

$n_{o}=$ collector optical efficiency

$Q_{u}=$ rate of useful energy delivered by collector
$T_{a}=$ ambient temperature.

(K)

$T_{r}=$ receiver temperature

$t_{i}=$ inside design temperature.

$t_{o}=$ outside design temperature

$U_{L}=$ heat loss coefficient

$\alpha=$ absorptivity

$a=$ average bond thickness

$\rho=$ mirror reflectance

$\tau=$ absorber transmittance

\section{Introduction}

Researches have been carried out to utilize the concentrated solar power in various fields. To utilize the solar energy different patterns of the collectors have been designed previously considering advantages as well as disadvantages [4]. From the previous literature survey it was found that parabolic concentrating collectors were developed to use the solar energy in an efficient way. Non tracking concentrator was also designed to avoid the handling of a tracking device $[1,3]$. To increase the performance innovative design was also proposed and modeled with reflectors without increasing the system cost. In that research work aluminum-polymerlaminated steel reflector for use in solar concentrators was evaluated with respect to its optical properties, durability, and reflector performance in solar thermal and photovoltaic systems [2]. In this paper a novel and innovative design of a solar collector which is basically Closed Environment Collector Trough (CECT) has been proposed. This novel CECT system lessens 
the rapid heat declination and improves the system overall efficiency. This paper mainly focuses the detail designing of the collector with accurate explanation.

\section{Methodology}

\subsection{Description of the system}

This paper proposes a new design of compound solar collector trough. The collector effectively combines Lenses, Reflectors and Closed Environment. The general structure of the trough is sphere shaped with two halves consisting a pipe at the center (Fig: 1). Different parts of the trough are labeled on the figure. A detailed description of different parts of the collector trough is given below.

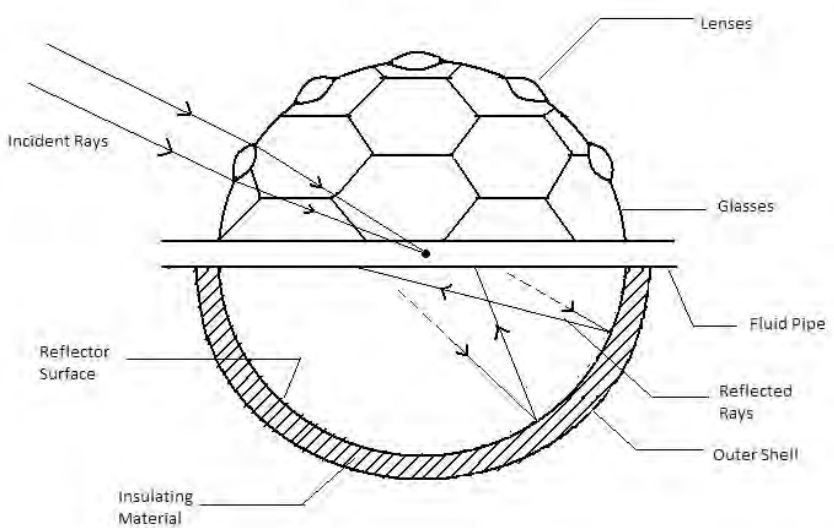

Figure 1: Closed Environment Collector Trough

The whole sphere shaped is main constructional shell of the system. It consists of two parts: Upper Shell and Lower Shell.

Lower Shell: Lower shell of the system is made of glazing metal (i.e. Aluminum). The outer surface is blackened and the inner surface is a reflector. There is another half sphere outside of this surface which is made of non-heat conductor material (i.e. asbestos). The inner diameter of the sphere is $40 \mathrm{c} . \mathrm{m}$ and outer diameter is $50 \mathrm{c.m}$. The space between the two surfaces is filled with cotton or rubber type materials (Fig: 2)

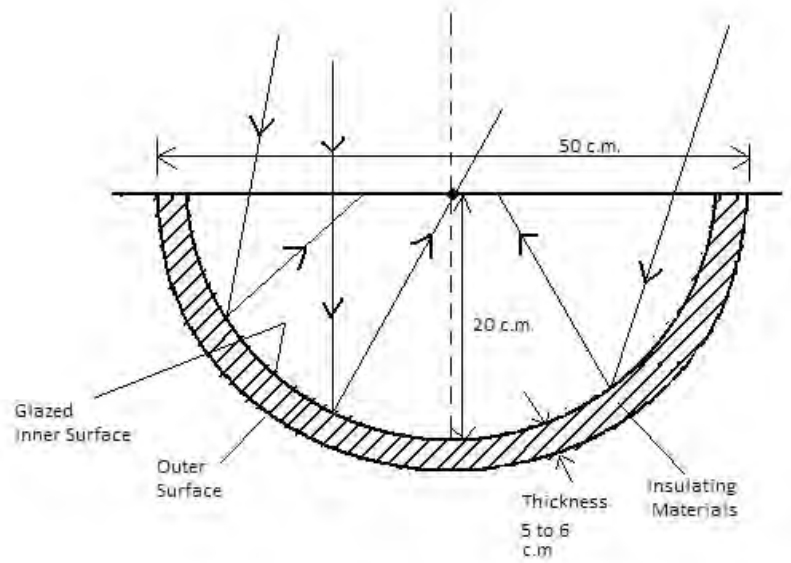

Figure 2: Lower shell 
Upper Shell: The upper shell of the system is made of metal three dimensional lattice of hexagonal shape (Fig: 3). These shapes will accommodate small pieces of mirrors, which will allow the visible light to enter into the chamber but will trap the longer wavelengths of the infrared re-radiation from the heated objects are unable to pass through the glasses. The trapping of the long wavelength radiation leads to more heating and a higher resultant temperature.

There will be five lenses, evenly distributed on the upper surface of the mirror section. The lenses will be $30^{\circ}$ apart from each other (Fig.4). The center lens will be at $90^{\circ}$ angle. These lenses will concentrate the sunlight in to center line (for the lenses the center line will be the focus plane) of the sphere.

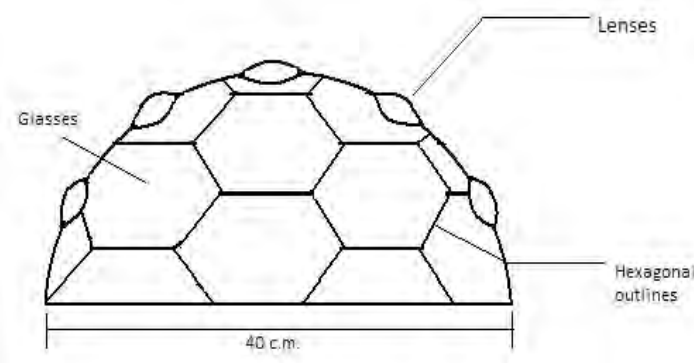

Figure 3: Upper Shell

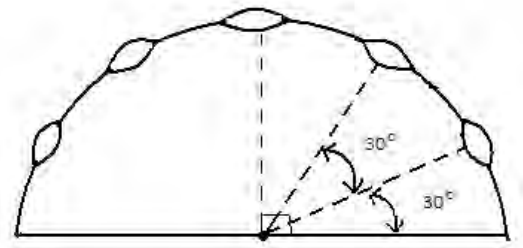

Figure 4: Arrangement of Lenses

Fluid Pipe: At the center of the sphere there will be a fluid carrying pipe. The fluid pipe will be consisted of two concentric pipes (Fig.5). The material of the outer pipe will be of transparent material (i.e. glass).The inner pipe will be metallic (i.e. Aluminum). The space between these two pipes will be evacuated. The inner pipe will carry the Heat Transfer fluid.

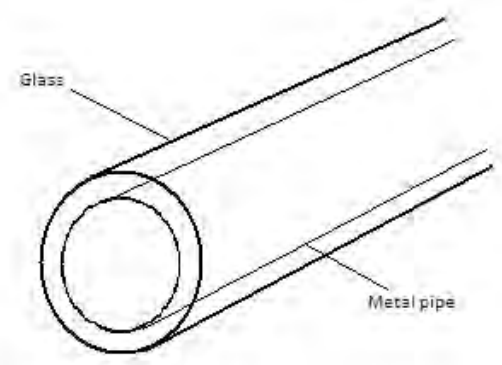

Figure 5: Fluid pipe

\subsection{Calculation of Sun's Position in Bangladesh}

To eliminate solar tracking mechanism effectively we have to calculate the sun's position throughout the year. Sun's position depends on local solar, elevation and azimuth angles. The equation to find the elevation and azimuth angles are given below:

Elevation $=\sin ^{-1}[\sin \gamma \sin \phi+\cos \gamma \cos \phi \cos H R A]$ 
Azimuth $=\cos ^{-1}\left[\frac{\sin \gamma \cos \phi-\cos \gamma \sin \phi \cos H R A}{\cos \alpha}\right]$

Where HRA is the Hour Angle

Sun's position throughout a year is given in Fig.6

As from the figure we can see that sun follows a specific path throughout the year, the solar tracking mechanism can be effectively eliminated by preparing a solar chart at a specific interval. The solar trough can be setup as shown in the Fig.7 and can be manually tracked daily basis [5].

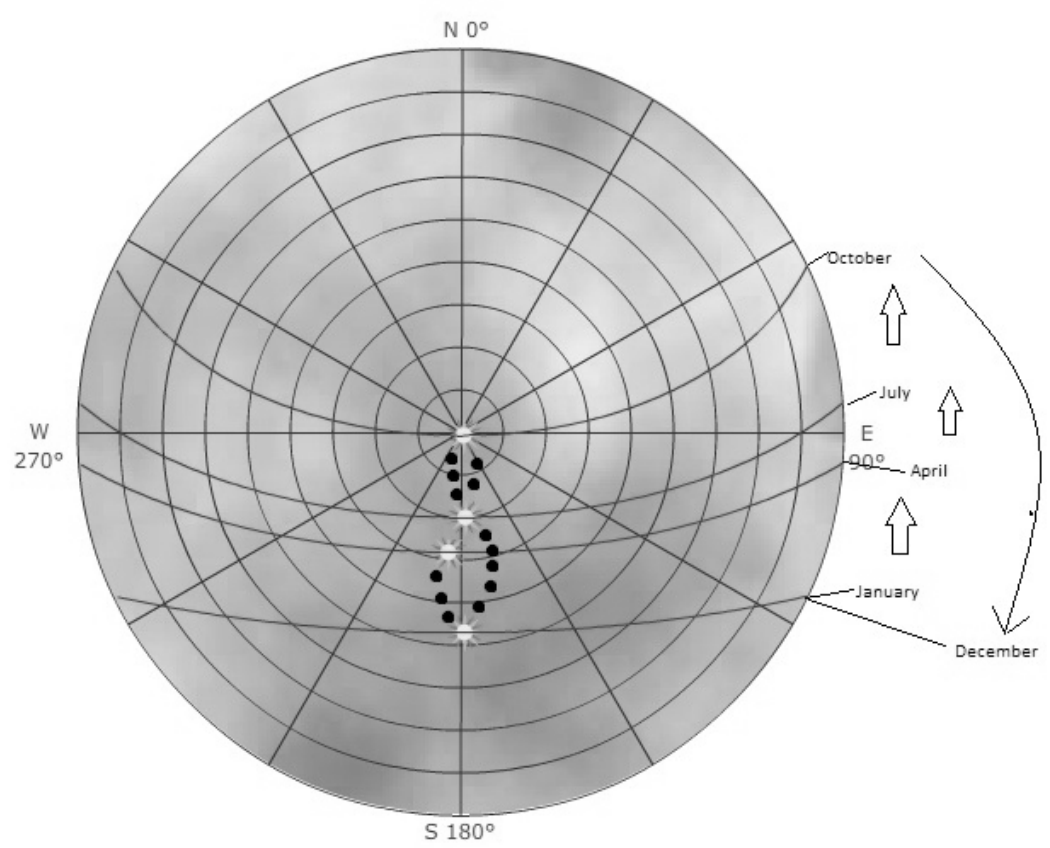

Figure 6: Sun's Position at 3 months interval. Dots represent the solar path

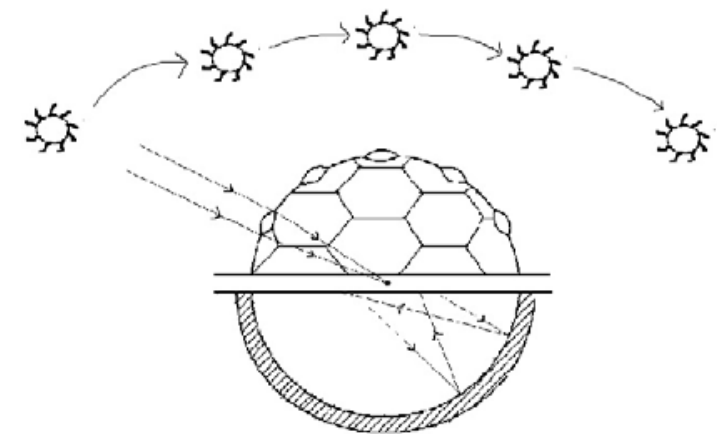

Figure 7: Collector position with respect to sun

\subsection{Calculation of Solar Insolation in Bangladesh}

Bangladesh is situated at $24^{\circ} 00^{\prime}$ North latitude and $90^{\circ} 00^{\prime}$ East longitude. At this position the amount of hours of sunlight each day throughout a year is shown in the following graph (Fig.8). The highest and the lowest intensity of direct radiation in $\mathrm{W} / \mathrm{m}^{2}$ are also shown in the 
Fig.9. This data shows that using solar collector for high temperature is feasible in Bangladesh.

\section{Calculation of useful energy delivered by the Collector}

The generation of heat inside the chamber will occur in three different methods:

Lens concentrator: The lenses placed on the surface of the upper half of the heat chamber will concentrate the sunlight directly in to the focus plane of the lenses which in this case is the center of the heat chamber where the fluid pipe will be placed Fig 10. This will directly heat up the heat transfer fluid.

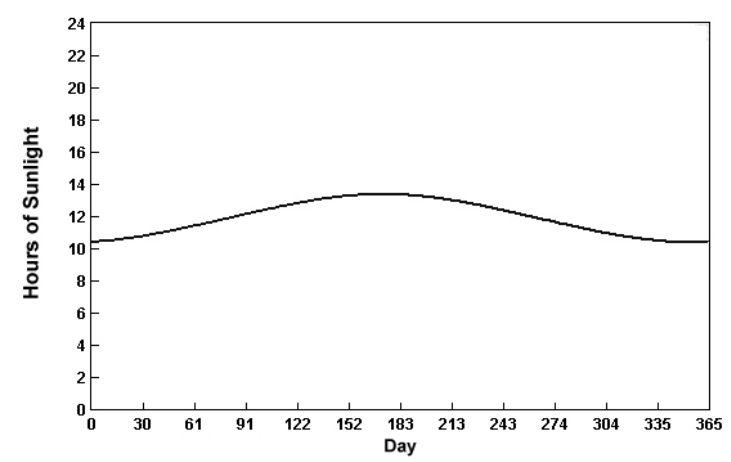

Figure 8: The amount of hours of sunlight in Bangladesh

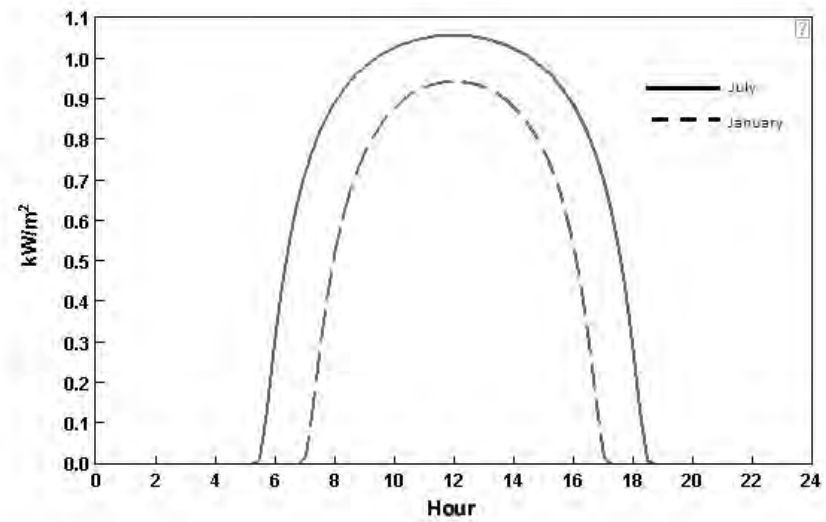

Figure 9: The highest and the lowest intensity of direct radiation in $\mathrm{W} / \mathrm{m}^{2}$

The heat (in joules) generated by the lenses could be found by the following equation:

$\mathrm{Q}=\mathrm{ms}\left(\mathrm{T}_{\mathrm{i}}-\mathrm{T}_{\mathrm{f}}\right)$

Where $Q$ is generated heat in joules, $m$ is mass of fluid in $K G, T_{i}$ is initial temperature of fluid in Kelvin and $\mathrm{T}_{\mathrm{f}}$ final temperature of fluid.

Reflector concentrator: This method will heat both the fluid pipe and the inner environment of the heat chamber. The concave bottom surface will act like a reflector mirror and most of the sunlight will be again concentrated on the pipe. This will result a thorough pipe heating (Fig. 11). 
The Green House: The hexagonal glasses of the upper half of the heat chamber will allow entering the visible light, but will trap the longer wavelengths of the infrared re-radiation from the heated objects. The trapping of the long wavelength radiation leads to more heating and a higher resultant temperature. This will keep the temperature of the chamber high.
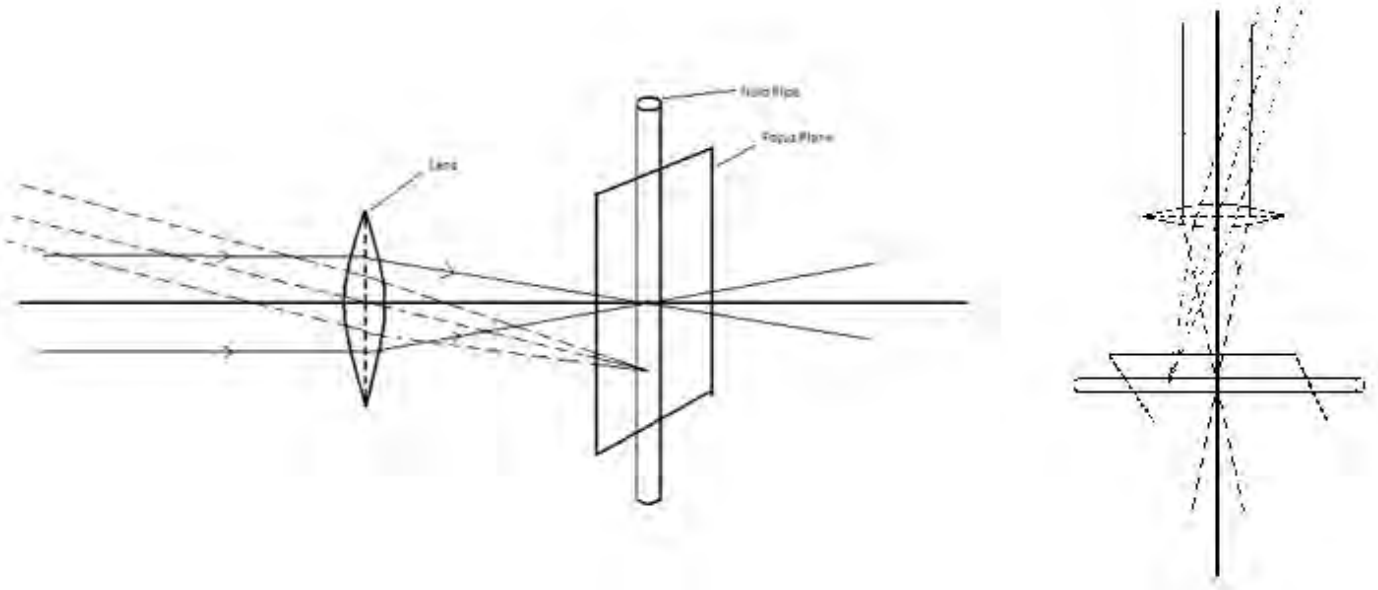

Figure 10: Lens and fluid pipe position
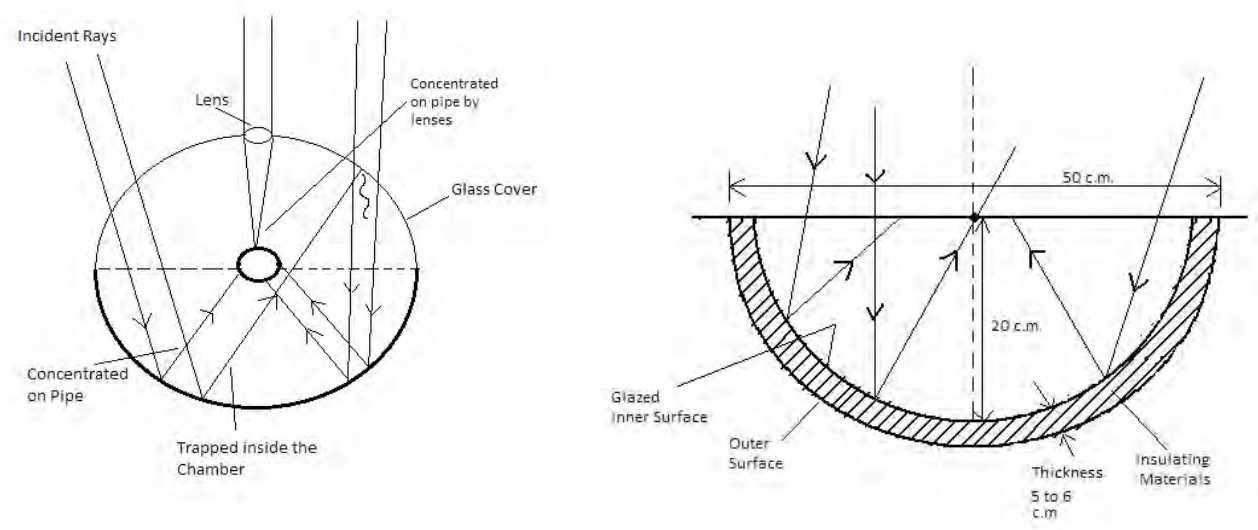

Figure 11: Front view and Section view of the collector

\subsection{Overall energy calculation}

The rate of useful energy delivered by the collector is governed by the following equation:

$Q_{u}=D_{s} n_{o} A_{a}-A_{r} U_{L}\left(T_{r}-T_{a}\right)$

As the system is a combination of Parabolic Collector Trough (PCT) the optical efficiency can be found from the PCT optical efficiency from the following equation:

$n_{o}=\rho \tau \varepsilon \partial\left[\left(1-A_{f} \tan (\varphi)\right) \cos (\varphi)\right]$

As the system use a green house the heat loss coefficient is determined by the following equation: 
$U_{L}=\left[\frac{A 1}{R 1}+\frac{A 2}{R 2}\right]\left(t_{i}-t_{o}\right) f_{w} f_{c}$

\section{Results}

A preliminary screening of the CECT is conducted in order to identify the best match of the load. The result is shown on a plot of efficiency as a function of the heat loss parameter in the following graph.

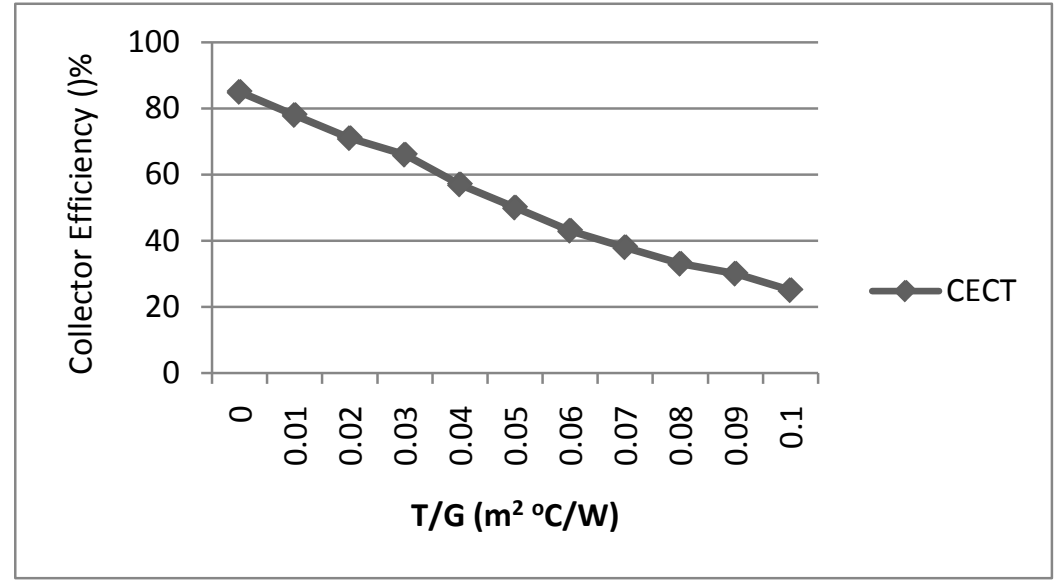

Figure 12: Collector efficiency

\section{Discussion}

Although Closed Environment Collector Trough is relatively a new concept, the system was expected to be more efficient than that of preliminary screening data. The future work should be based on the improvement of current design and optical as well as thermal optimization.

\section{References}

[1] H. E. Imadojemu, Concentrating parabolic collectors: A patent survey Energy Conversion and Management, Volume 36, Issue 4, April 1995, Pages 225-237

[2] Maria Brogren, Anna Helgesson, Björn Karlsson, Johan Nilsson, Arne Roos, Optical properties, durability, and system aspects of a new aluminium-polymer-laminated steel reflector for solar concentrators, Solar Energy Materials and Solar Cells, Volume 82, Issue 3, 15 May 2004, Pages 387-412

[3] A. Thomas, Solar steam generating systems using parabolic trough concentrators Energy Conversion and Management, Volume 37, Issue 2, February 1996, Pages 215-245

[4] Soteris A. Kalogirou, Solar thermal collectors and applications Progress in Energy and Combustion Science, Volume 30, Issue 3, 2004, Pages 231-295

[5] http://pvcdrom.pveducation.org/SUNLIGHT/SUNCALC.HTM 